\title{
Throughput Optimal Communication Strategy for Wireless Random Access Channel
}

\author{
Harpreet S. Dhillon, Howard C. Huang, Harish Viswanathan and Reinaldo A. Valenzuela
}

\begin{abstract}
We consider a wireless time-slotted random access channel where user arrivals are characterized by a Poisson process. Each user comes with a fixed payload, which has to be transmitted in the slot in which it arrives. If the transmission is successful, the user leaves the system, else it is dropped. The receiver and users are assumed to have knowledge of the arrival rate $\lambda$, but they are not aware of the actual number of users simultaneously attempting to communicate during a given time slot. In contrast to a conventional slotted ALOHA-based strategy where the channel is partitioned into orthogonal subchannels and each user communicates on a randomly chosen subchannel, we propose a novel strategy whereby users transmit simultaneously over the entire channel resource and the receiver jointly decodes the transmissions. Under the proposed strategy, neither users nor the receiver have prior knowledge of the active user set. Our analysis concretely demonstrates that the proposed strategy is optimal in terms of maximizing the average throughput among all uncoordinated strategies. Numerical results show that the proposal provides an order of magnitude throughput improvement compared to slotted ALOHA in a single-cell environment under a $10 \%$ maximum outage constraint.
\end{abstract}

\section{INTRODUCTION}

Random access is a common strategy for multiple users attempting to communicate to a single receiver over shared wireless channel resources. For example, it is used in wireless local area networks (LANs) under IEEE 802.11 standards for payload transmission and in cellular networks under wideband code division multiple access (WCDMA) and long term evolution (LTE) standards for initiating uplink transmissions [1]. Random access becomes especially attractive for data transmission when the payloads are small, e.g., for machine-tomachine (M2M) communications where the payloads are of the order of a few hundred bytes [2], [3].

A common model for random access is the slotted ALOHA network, where the time domain is partitioned into slots and users randomly transmit at a fixed rate during a given time slot. Packet collisions occur when more than one user transmits during a given slot. This model can be generalized further by partitioning each time slot into orthogonal frequency resources, where each user chooses a frequency resource at random. For example in cellular random access, the channel is partitioned into orthogonal spreading codes, and collisions occur when more than one user chooses a given code [1].

In contrast to this network-based approach to random access communication, there has been relatively little information

H. S. Dhillon is with WNCG, the University of Texas at Austin, USA. Email: dhillon@utexas.edu. H. C. Huang, H. Viswanathan and R. A. Valenzuela are with Bell Labs, Alcatel-Lucent, NJ. Email: \{howard.huang, harish.viswanathan, reinaldo.valenzuela\}@alcatel-lucent.com. theory-based analysis that accounts for the noise and interference caused by simultaneous transmissions [4]. One recent exception is [5], which provides a rigorous information theoretic framework and characterizes the achievable rate region within a guaranteed gap of the optimal region.

Our study in this paper differs from [5] in two important ways. First, we assume all users transmit with a fixed rate (spectral efficiency) $\mathcal{R}$ bps $/ \mathrm{Hz}$ as derived from the fixed payload size (in bits), the time slot duration (in seconds) and bandwidth (in $\mathrm{Hz}$ ). Second, we incorporate random arrivals, where the number of users with data to transmit in each time slot are characterized by a Poisson process with mean $\lambda$. We propose a novel strategy for maximizing the average throughput, defined as the product of $\mathcal{R}, \lambda$, and the probability of success for a given user.

Under our proposal, each user with data decides to transmit independently of the other users and the channel gains with certain fixed probability, which is the same for all the users. A transmitting user, also termed as an active user, chooses a codebook at random from a set of Gaussian codebooks known to all users and the receiver. An active user then transmits a preamble sequence, uniquely associated with the chosen codebook, followed by the encoded message. In contrast to the cellular random access model, where users transmit over randomly chosen orthogonal frequency resource, each active user transmits over the entire bandwidth under proposed strategy. The receiver first detects the presence of active codebooks using the preambles and then jointly decodes them. During a given time slot, the transmissions are successful if the rate vector, corresponding to the set of active users, lies within the multiple access channel (MAC) capacity region defined by the users' received signal-to-noise ratios (SNRs).

The paper is organized as follows. In Section II, we describe the system model of a single cell wireless network. In Section III, we formulate the problem of maximizing throughput in an uncoordinated, random access channel, and we describe our proposed optimal strategy. We also formulate the throughput of a conventional random access strategy based on frequency division multiple access (FDMA). In Section IV, we show numerical results comparing the outage and throughput performance of the optimal and FDMA strategies.

\section{SYSTEM MODEL}

Consider the uplink of a single cell system in which the base station is located at the origin and the users are uniformly distributed around it in a circular disk of radius $r_{o}$. Although slightly idealistic for cellular networks, this scenario 


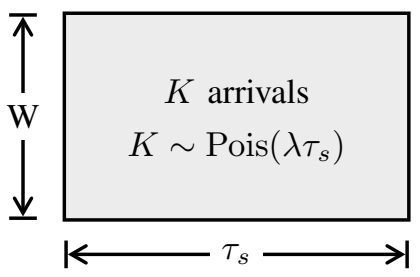

Fig. 1. A typical time-frequency resource "slice" with $K$ arrivals.

is still relevant for a variety of applications, e.g., it is one of the simulation scenarios in 3GPP model to study M2M communication in cellular networks [6]. The users transmit such that the number of arrivals at the base station can be modeled as a Poisson process with rate $\lambda$ arrivals per second. Time is divided into slots of duration $\tau_{s}$ secs and total available bandwidth is $W \mathrm{~Hz}$. Each such slot acts as a "resource slice" as shown in Fig. 1. The number of users with data to transmit in any given slot is denoted by $K$, which is Poisson distributed with mean $\lambda \tau_{s}$. For multiple access, we consider uncoordinated transmission, where the base station does not play any role in scheduling users, i.e., it neither decides the set of transmitting users nor their exact scheduling over timefrequency resources.

We assume that each user enters the system with a deadline of $\tau_{s}$ secs, i.e., it transmits over a single time slot and leaves the system upon successful completion of the data transfer. If the transmission is not successful, the packet is dropped and is said to be in outage. Throughout this paper, we restrict our attention to transmission strategies in which all the users transmit at the same average rate $\mathcal{R}$ over the duration $\tau_{s}$ whenever they transmit. Although it may be advantageous to have different users transmitting at different rates, such strategies are outside the scope of this paper. Each user is assumed to have a maximum transmit power constraint of $P_{\max }$. The uplink channel is modeled as a combination of power-law pathloss, large scale shadowing and small scale fading effects. Therefore, the received power at the base station from a device located at distance $r$ is

$$
P_{r}=P_{t} \mathcal{X} h G r^{-\gamma}
$$

where $P_{t} \leq P_{\max }$ is the transmit power, $\mathcal{X}$ is a log-normal random variable modeling shadowing gain with standard deviation $\sigma \mathrm{dB}, h \sim \exp (1)$ models small scale channel gain due to Rayleigh fading, $G$ is the direction based antenna gain, $\gamma$ is the pathloss exponent, and the device index is suppressed for simplicity of notation. To study the composite effect of all these link budget parameters, we define the reference SNR $\mu$ as the average received SNR from a device transmitting at maximum power $P_{\max }$ over bandwidth $W$ located at cell edge, i.e., at distance $r_{o}$ from the base station. Therefore, the received SNR $\mu_{r}$ at the base station from a device located at distance $0<r \leq r_{o}$ and transmitting over a bandwidth $W_{\mathcal{N}} \leq W$ can be expressed in terms of $r$ as

$$
\mu_{r}=\frac{W}{W_{\mathcal{N}}} \frac{P_{t}}{P_{\max }} \mu \mathcal{X} h\left(\frac{r}{r_{o}}\right)^{-\gamma},
$$

where the factor $\frac{W}{W_{\mathcal{N}}}$ accounts for the difference in the noise
TABLE I

\begin{tabular}{|c|c|}
\hline Notation & Description \\
\hline $\bar{W}$ & Total bandwidth in $\mathrm{Hz}$ \\
\hline$\tau_{s}$ & Slot duration in secs \\
\hline$\lambda$ & Rate of new arrivals at the base station \\
\hline$g_{k}$ & The effective channel gain of $k^{t h}$ device \\
\hline$P_{\max }$ & Maximum power constraint \\
\hline$\mu$ & Reference SNR \\
\hline$K ; K_{s}$ & $\begin{array}{l}\text { Number of arrivals in each slot; number of successful } \\
\text { transmissions }\end{array}$ \\
\hline $\mathcal{R} ; \mathcal{S}$ & Maximum common rate; maximum throughput \\
\hline$\epsilon$ & Outage probability \\
\hline$\Theta$ & $\begin{array}{l}\text { Transmission probability in the optimal uncoordinated } \\
\text { strategy }\end{array}$ \\
\hline $\mathbb{Z}_{k_{1}}^{k_{2}}$ & $\left\{k_{1}, k_{1}+1, \ldots, k_{2}\right\} \subseteq \mathbb{Z}$ for $k_{1} \leq k_{2}$ \\
\hline$\left\{x_{k}\right\}_{k_{1}}^{k_{2}}$ & $\left\{x_{k_{1}}, x_{k_{1}+1}, \ldots x_{k_{2}}\right\}$ for $k_{1} \leq k_{2}$ \\
\hline
\end{tabular}

NOTATION SUMMARY

power due to the difference in the bandwidths for which $\mu_{r}$ and $\mu$ are defined. This will be helpful in analyzing the uncoordinated FDMA strategy in the next section. Now defining the effective channel gain as $g=\mathcal{X} h\left(\frac{r}{r_{o}}\right)^{-\gamma}$, the expression for received SNR $\mu_{r}$ can be further simplified to

$$
\mu_{r}=\frac{W}{W_{\mathcal{N}}} \frac{P_{t}}{P_{\max }} \mu g
$$

For tractability, we assume capacity achieving codes. Please note that the effect of finite block length, especially important when the payloads are small, e.g., in M2M communications, can be easily incorporated by means of an SNR gap. Interested readers can refer to [7] for more details. For a user transmitting over time $\tau_{\mathcal{N}} \leq \tau_{s}$, bandwidth $W_{\mathcal{N}} \leq W$, and with slight overloading of $\mu_{r}$ to denote received signal-to-interferenceplus-noise ratio (SINR), the rate (or more precisely, the spectral efficiency) achieved by the user over a particular timefrequency resource slice can be expressed as

$$
\mathcal{R}=\frac{\tau_{\mathcal{N}}}{\tau_{s}} \frac{W_{\mathcal{N}}}{W} \log _{2}\left(1+\mu_{r}\right) \text { bps/Hz }
$$

The received SINR depends upon the decoding strategy as discussed in the next section. For the special case of FDMA, where a user with channel gain $g$ transmits at $P_{\max }$ over $\frac{W}{B}$ $\mathrm{Hz}$, the rate achieved is

$$
\mathcal{R}=\frac{1}{B} \log _{2}\left(1+\mu_{r}\right) \stackrel{(a)}{=} \frac{1}{B} \log _{2}(1+B \mu g),
$$

where the pre-log factor of $\frac{1}{B}$ comes from (4) and (a) follows from (3). For ease of notation, we denote the set of integers from $k_{1}$ to $k_{2}>k_{1}$ by $\mathbb{Z}_{k_{1}}^{k_{2}}=\left\{k_{1}, k_{1}+1, \ldots, k_{2}\right\} \subseteq \mathbb{Z}$. Similarly, any general sequence $\left\{x_{k_{1}}, x_{k_{1}+1}, \ldots x_{k_{2}}\right\}$ for $k_{1} \leq$ $k_{2}$ is denoted by $\left\{x_{k}\right\}_{k_{1}}^{k_{2}}$. The notation used in this paper is summarized in Table I for quick reference.

\section{UnCOORDINATED MULTIPLE ACCESS}

This is the main technical section of the paper where we study the maximum throughput for uncoordinated strategies. We first formulate the throughput maximization problem in terms of outage probability, common rate and the user arrival rate. We then propose a novel multiuser detection strategy and establish its optimality for uncoordinated transmission. 
To compare the optimal results with a realistic baseline, we also formulate the throughput maximization problem for uncoordinated FDMA.

\section{A. Problem Formulation}

We begin this discussion by introducing the main metric of interest for this work, which is the average throughput $\mathcal{S}$, for a given arrival rate $\lambda$ and a given transmission strategy that captures the average number of successfully transmitted bits per unit time per unit bandwidth. For a slot with $K$ arrivals, out of which $K_{s}$ succeed, the average throughput $\mathcal{S}$ can be expressed as

$$
\mathcal{S}(\lambda, \mu, \mathcal{R})=\frac{1}{\tau_{s}} \mathbb{E}\left[K_{s}\right] \mathcal{R}
$$

where $\mathcal{R}$ denotes the common rate of each device for the given transmission strategy, $\mu$ is the reference SNR and $K \sim$ $\operatorname{Pois}\left(\lambda \tau_{s}\right)$ by assumption. The rest of the arrivals are dropped and the corresponding users are said to be in outage. Due to the packet deadline of $\tau_{s}$, these dropped packets cannot be considered for a future transmission. Now assuming $\mathcal{T}_{i}^{(s)}$ denotes the event that the $i^{t h}$ packet, for $i \in \mathbb{Z}_{0}^{K}$, succeeds, the number of successful transmissions $K_{s}$ can be expressed in terms of $K$ as

$$
K_{s}=\sum_{i=1}^{K} \mathbf{1}\left(\mathcal{T}_{i}^{(s)}\right)
$$

where $\mathbf{1}(\mathcal{E})$ is an indicator function that takes value 1 when event $\mathcal{E}$ occurs and 0 otherwise. Using (7), $\mathbb{E}\left[K_{s}\right]$ can be derived for a given arrival rate $\lambda$ as follows:

$$
\begin{aligned}
\mathbb{E}\left[K_{s}\right] & =\mathbb{E}_{K} \mathbb{E}\left[\sum_{i=1}^{K} \mathbf{1}\left(\mathcal{T}_{i}^{(s)}\right) \mid K\right] \\
& \stackrel{(a)}{=} \mathbb{E}_{K}\left[K \mathbb{P}\left(\mathcal{T}_{i}^{(s)}\right)\right] \\
& =(1-\epsilon) \mathbb{E}[K],
\end{aligned}
$$

where $(a)$ follows from the linearity of inner expectation. Thus, the outage probability can be expressed in terms of $K$ and $K_{s}$ as

$$
\epsilon=1-\frac{\mathbb{E}\left[K_{s}\right]}{\mathbb{E}[K]}
$$

using which the average throughput $\mathcal{S}$ can be expressed as

$$
\mathcal{S}(\lambda, \mu, \mathcal{R})=\frac{\mathbb{E}[K]}{\tau_{s}} \mathcal{R}(1-\epsilon)=\lambda \mathcal{R}(1-\epsilon) .
$$

Given a maximum outage constraint $\epsilon_{\max }$, the throughput maximization problem can now be formulated as

$$
\begin{array}{ll}
\max _{\mathcal{R}} & \lambda \mathcal{R}(1-\epsilon) \\
\text { s.t. } & \epsilon \leq \epsilon_{\max }
\end{array} .
$$

We will henceforth refer to the maximum throughput corresponding to the solution of the above optimization problem as the rate-maximized throughput. We now remark on the solution of this optimization problem below.

Remark 1 (Solution procedure). In addition to the arrival rate and the choice of multiple access strategy, the outage probability $\epsilon$ is also a function of the common rate of the users. Therefore, for a given arrival rate $\lambda$, there is a maximum common rate $\mathcal{R}$ corresponding to each value of outage probability $\epsilon$. As discussed for uncoordinated optimal and FDMA strategies in this section, it is, in principle, possible to characterize this relationship analytically. However, the form of this relationship is such that it does not lead to a closed form expression for the maximum throughput. Therefore, we have to resort to the numerical solution for the above optimization problem, which is straightforward once the relationship between the maximum common rate $\mathcal{R}$ and outage probability $\epsilon$ is established. Characterizing this relationship for the optimal and FDMA strategies is our goal for the rest of this subsection.

To highlight the fact that the outage probability is a function of transmission strategy $\Pi$, arrival rate $\lambda$, reference SNR $\mu$ and common rate $\mathcal{R}$, we let $\mathcal{E}^{\Pi}(\lambda, \mu, \mathcal{R})$ denote the outage function as defined by (11). As remarked above, for a desired outage level $\epsilon$, the maximum common rate $\mathcal{R}^{*}(\lambda, \epsilon, \mu)$ can be determined as follows:

$$
\mathcal{R}^{*}(\lambda, \epsilon, \mu)=\arg \max _{\mathcal{R}}\left[\mathcal{E}^{\Pi}(\lambda, \mu, \mathcal{R}) \leq \epsilon\right],
$$

for which we need to characterize outage function $\mathcal{E}^{\Pi}(\lambda, \mu, \mathcal{R})$, which is done next.

\section{B. Optimal Uncoordinated Multiple Access}

Using (11), the outage function for the optimal uncoordinated strategy can be expressed as:

$$
\begin{aligned}
\mathcal{E}^{\Pi_{U O}}(\lambda, \mu, \mathcal{R}) & =\min _{\Pi_{U O}}\left[1-\frac{\mathbb{E}_{K,\left\{g_{k}\right\}}\left(K_{s}\right)}{\mathbb{E}(K)}\right] \\
& =1-\frac{\max _{\Pi_{U O}} \mathbb{E}_{K,\left\{g_{k}\right\}}\left(K_{s}\right)}{\mathbb{E}(K)} \\
& =1-\frac{\max _{\Pi_{U O}} \mathbb{E}_{K,\left\{g_{k}\right\}}\left(K_{s}\right)}{\lambda \tau_{s}},
\end{aligned}
$$

where $K$ is the number of arrivals in a particular slot and $K_{s}$ is the number of these arrivals that succeed. The goal now is to derive $\mathbb{E}\left[K_{s}\right]$, which involves a novel multiuser decoding strategy as discussed in the next theorem. The details of the proposed strategy appear in the achievability part of the proof.

Theorem 1. The mean number of users that successfully communicate at rate $\mathcal{R}$ during a given time slot in the optimal uncoordinated transmission strategy is given by

$$
\mathbb{E}\left[K_{s}\right]=\max _{\Theta} \lambda \tau_{s} \Theta \mathbb{E}_{\Omega}\left[\max _{|\mathcal{L}|} \frac{|\mathcal{L}|}{\Omega} \mathbb{P}\left(\mathcal{C}\left(P_{\max }\right)\right)\right],
$$

where $\Omega \sim \operatorname{Pois}\left(\lambda \tau_{s} \Theta\right)$ and the event $\mathcal{C}\left(P_{\max }\right)$ is defined as

$$
\left\{|\widetilde{\mathcal{L}}| \mathcal{R} \leq \log _{2}\left(1+\frac{\mu \sum_{i \in \widetilde{\mathcal{L}}} g_{i}}{1+\mu \sum_{m \in \mathcal{T}-\mathcal{L}} g_{m}}\right), \forall \widetilde{\mathcal{L}} \subseteq \mathcal{L}\right\},
$$

where $\mathcal{L} \subset \mathcal{T}$ and $\mathcal{T}$ is the set of users transmitting in the given slot, with $|\mathcal{T}|=\Omega$.

The proof of this theorem involves both converse and achievability parts, discussed in detail below. 
Proof: (Converse) To prove the converse, consider any strategy $\Pi_{U}$ for uncoordinated transmission that governs: i) the decision to transmit, and ii) the power level of a user which has data to transmit in any given slot independent of total number of users $K$ in that slot and their channel gains $\left\{g_{k}\right\}_{1}^{K}$. Let $\widetilde{s}(n)$ be the indicator for whether user $n$ transmits, i.e., $\widetilde{s}(n)=1$ when user $n$ transmits and $\widetilde{s}(n)=0$ otherwise. Let $P_{n} \leq$ $P_{\max }$ be the transmit power of user $n$ that is transmitting. Note that for any uncoordinated transmission strategy, $\widetilde{s}(n)$ and $P_{n}$ are independent of $K,\left\{g_{k}\right\}_{1}^{K}$ and independent of each other. Denote the set of active users by $\mathcal{T}$, i.e.,

$$
\mathcal{T}=\{n: \widetilde{s}(n)=1\} .
$$

Denote the common rate by $\mathcal{R}$ and suppose a subset $\mathcal{L} \subset \mathcal{T}$ of users' messages are successfully decoded by the receiver. Then from the MAC theorem [8], we require that

$$
l \mathcal{R} \leq \log _{2}\left(1+\sum_{i \in \widetilde{\mathcal{L}}} \frac{\frac{P_{i}}{P_{\max }} \mu g_{i}}{1+\sum_{m \in \mathcal{T}-\mathcal{L}} \frac{P_{m}}{P_{\max }} \mu g_{m}}\right), \forall \widetilde{\mathcal{L}} \subset \mathcal{L},
$$

where $l=|\widetilde{\mathcal{L}}|$. For ease of notation, we denote this event by $\mathcal{C}\left(\left\{P_{n}\right\},\left\{g_{n}\right\}, \mu, \mathcal{L}, \mathcal{T}\right)$. In the rest of the proof, we will use the short hand notation $\mathcal{C}\left(\left\{P_{n}\right\}\right)$, with the understanding that it does depend upon other parameters but they are not important for the following arguments. The average number of users that are successfully decoded can now be upper bounded by

$$
\mathbb{E}\left[K_{s}\right] \leq \max _{\left\{P_{n}\right\}} \mathbb{E}_{K, \widetilde{s}(n)} \mathbb{E}_{\left\{g_{n}\right\}}\left[\max _{|\mathcal{L}|}|\mathcal{L}| \mathbf{1}\left(\mathcal{C}\left(\left\{P_{n}\right\}\right)\right) \mid K,\{\widetilde{s}(n)\}\right]
$$

$$
\begin{aligned}
& \stackrel{(a)}{\leq} \max _{P} \mathbb{E}\left[\mathbb{E}\left[\max _{|\mathcal{L}|}|\mathcal{L}| \mathbf{1}(\mathcal{C}(P)) \mid K,\{\widetilde{s}(n)\}\right]\right] \\
& \stackrel{(b)}{\leq} \mathbb{E}\left[\mathbb{E}\left[\max _{|\mathcal{L}|}|\mathcal{L}| \mathbf{1}\left(\mathcal{C}\left(P_{\max }\right)\right) \mid K,\{\widetilde{s}(n)\}\right]\right] \\
& \stackrel{(c)}{=} \mathbb{E} \mathbb{E}\left[\frac{\sum_{n=1}^{K} \widetilde{s}(n)}{\Omega} \max _{|\mathcal{L}|}|\mathcal{L}| \mathbf{1}\left(\mathcal{C}\left(P_{\max }\right)\right) \mid K,\{\widetilde{s}(n)\}\right]
\end{aligned}
$$

$$
\begin{aligned}
& \stackrel{(d)}{=} \mathbb{E}\left[\sum_{n=1}^{K} \widetilde{s}(n)\right] \mathbb{E}_{\Omega,\left\{g_{n}\right\}}\left[\max _{|\mathcal{L}|} \frac{|\mathcal{L}|}{\Omega} \mathbf{1}\left(\mathcal{C}\left(P_{\max }\right)\right)\right] \\
& =\lambda \tau_{s} \Theta \mathbb{E}_{\Omega}\left[\max _{|\mathcal{L}|} \frac{|\mathcal{L}|}{\Omega} \mathbb{E}_{\left\{g_{n}\right\}} \mathbf{1}\left(\mathcal{C}\left(P_{\max }\right)\right)\right] \\
& =\lambda \tau_{s} \Theta \mathbb{E}_{\Omega}\left[\max _{|\mathcal{L}|} \frac{|\mathcal{L}|}{\Omega} \mathbb{P}\left(\mathcal{C}\left(P_{\max }\right)\right)\right]
\end{aligned}
$$

where $(a)$ follows from the fact that the transmit power is independent of all the other variables, including channel gains of the respective user, which means that "optimal" value will be the same for all the users, $(b)$ follows from the fact that the following function

$$
\sum_{i \in \mathcal{L}} \frac{\frac{P}{P_{\max }} \mu g_{i}}{1+\sum_{m \in \widetilde{\mathcal{T}}-\mathcal{L}} \frac{P}{P_{\max }} \mu g_{m}},
$$

is an increasing function of $P$ for given set of channel gains, (c) follows by simply setting $\Omega=\sum_{n=1}^{K} \widetilde{s}(n)$, which is a
Poisson distributed random variable with mean $\lambda \tau_{s} \Theta$, and $(d)$ follows from the independence of $\widetilde{s}(n)$ from all other random variables. This completes the proof of the converse.

(Achievability) We now present the achievability proof, i.e., show that the above upper bound on the average number of successfully decodable users is achievable as the number of channel symbols per slot goes to infinity. First, pick a set of $\bar{N}$ code books for the AWGN MAC, each with $2^{\mathcal{R}}$ code words of length $\bar{n}$. The code words can be picked at random from the typical set of Gaussian random input distribution with transmit power $P_{\max }$ as is usually done in the proof of the MAC coding theorem [8]. Observe that the code books for each user are selected independently of the other users and the SNR. Furthermore, the code book is specifically not dependent on the channel gain except for the number of code words, which is governed by the rate $\mathcal{R}$. Thus a combination of such independently chosen code books can form the code book of the MAC.

At the beginning of each transmission, a preamble Gaussian sequence of length $q$ that is unique to that code book is transmitted to help the receiver detect which code books are being transmitted. All the code words of the same code book will have the same sequence while the different code books will have distinct sequences. The sequences can be drawn at random from a Gaussian random variable with variance $P_{\max }$.

As before, suppose there are $K$ users who have data to transmit in a given slot. In our strategy, each user will decide to transmit with probability $\Theta$. Recall that the total number of transmitting users is denoted by $\Omega$. When a user decides to transmit, it will pick one of the code books from $\bar{N}$ code books at random and map the message to one of the code words in the chosen code book in the usual way and first transmit the preamble followed by the code word.

The collision probability $\mathcal{P}_{c}$ that two or more transmitting users pick the same code book when $\Omega$ users attempt to transmit is given by

$$
\mathcal{P}_{c}(\Omega)=1-\left(1-\frac{1}{\bar{N}}\right)^{\Omega-1},
$$

which is proved in Lemma 1 of [9]. Since $\Omega$ is a Poisson random variable, there exists $\Omega_{\max }$ such that $\mathbb{P}\left\{\Omega>\Omega_{\max }\right\}<$ $\delta^{\prime} / 2$. By picking $\bar{N}$ large enough so that $\mathcal{P}_{c}\left(\Omega_{\max }\right) \leq \delta^{\prime} / 2$, we can ensure that the overall collision probability is $\mathcal{P}_{c} \leq \delta^{\prime}$.

Now consider only the case when the transmitting users have picked distinct code books. We propose a decoding strategy in which the receiver first detects which code books are in use, i.e, code words from which code books are transmitted in that slot, and then proceed to decode the code words. With a simple correlator detector, the receiver can detect the presence of each of the possible code books. The total detection error - detecting positively a code book that is not used as well as missing a code book that is in use - can be bounded by

$$
\begin{gathered}
\mathcal{P}_{d} \leq \bar{N} \exp \left(-q \Phi\left(\frac{\mu g_{\min }}{1+(\bar{N}-1) \mu g_{\max }}\right)\right)+ \\
\bar{N}\left(\mathbb{P}\left(g<g_{\text {min }}\right)+\mathbb{P}\left(g>g_{\max }\right)\right),
\end{gathered}
$$


where we bounded the individual detection error probabilities assuming the worst case channel scenario, i.e., the interferers have some large channel gain while the desired signal being detected has a small channel gain. Observe that it is possible to first pick $g_{\min }$ and $g_{\max }$ so that $\bar{N}\left(\mathbb{P}\left(g<g_{\min }\right)+\mathbb{P}\left(g>g_{\max }\right)\right) \leq \delta^{\prime \prime} / 2$ and then pick $q$ sufficiently large so that $\mathcal{P}_{d} \leq \delta^{\prime \prime}$. Furthermore, since $q$ is fixed relative to block length, the loss in capacity because of the preamble can be made arbitrarily small. The resulting loss in transmission rate can thus be made negligible. Thus we can assume that the receiver knows which code books are in use. Note that since the channels are static the receiver can similarly first estimate the channel accurately from the preamble and then apply the joint typicality test for the known channels. We will assume that the channel is estimated without errors observing that the increase in decoding error probability because of channel estimation errors can be shown to be arbitrarily small when $q$ goes to infinity.

Once the channels are determined, the receiver tries to evaluate which subset of code books from the detected code books can actually be decoded treating the rest as noise. To this end, the receiver can compute the rate region for decoding all possible subsets of code books detected and pick the largest set of users for which the rate $\mathcal{R}$ is achievable. In other words, the receiver can find the subset $\mathcal{L}$ of the set of code books $\mathcal{T}$ detected to be in use. Thus, the receiver will attempt to decode the maximal set of code words from the code books for which

$$
|\widetilde{\mathcal{L}}| \mathcal{R} \leq \log _{2}\left(1+\sum_{i \in \widetilde{\mathcal{L}}} \frac{\mu g_{i}}{1+\sum_{m \in \mathcal{T}-\mathcal{L}} \mu g_{m}}\right), \forall \widetilde{\mathcal{L}} \subset \mathcal{L}
$$

From the MAC theorem, we know that whenever the above condition is satisfied then the decoding error,

$$
\mathcal{P}_{e} \leq \delta^{\prime \prime \prime}(\bar{n})
$$

where $\delta^{\prime \prime \prime} \rightarrow 0$ as $\bar{n} \rightarrow \infty$.

Thus as $\bar{n} \rightarrow \infty$, the combined error probability $\mathcal{P}_{c}+\mathcal{P}_{d}+$ $\mathcal{P}_{e} \rightarrow 0$ whenever the rate constraint (32) is met. Thus the bound on RHS of (28) is achievable.

Remark 2 (Numerical computation of (18)). The mean number of successful users given by (18) can be numerically computed via Monte Carlo simulations, where the expectation with respect to $\Omega$ corresponds to the Poisson arrival process of the users (with mean $\lambda \tau_{s} \Theta$ ) whose channels are obtained by uniformly placing them on a disk of radius $r_{o}$.

\section{Uncoordinated FDMA with Equal Allocation}

Assume that the bandwidth is partitioned into $B \geq K$ equal subbands. Using (11), we derive the outage function for uncoordinated FDMA with equal bandwidth allocation. We assume that the users choose one of the $B$ subbands randomly. Since the aggregate arrivals are modeled as Poisson and each user chooses a subband randomly, the arrival process in each subband can also be modeled as Poisson with an appropriately scaled arrival rate [10]. If a subband is chosen by more than two users, the transmission of all those users is assumed to be unsuccessful. Note that this corresponds to a worst case scenario, because in practice one of these users may still have a strong enough channel to ensure successful transmission even after treating the interference as noise. Nevertheless, under these assumptions, the transmission is successful for a given common rate only when the following two conditions are met: i) the user under consideration is the only one to choose a particular subband, and ii) its channel is sufficiently strong to achieve common rate $\mathcal{R}$ over the chosen band. Using (5), the rate of the user with channel gain $g_{k}$ transmitting over bandwidth $B$ is

$$
\widetilde{\mathcal{R}}_{f}\left(g_{k}, \mu, B\right)=\frac{1}{B} \log _{2}\left(1+B \mu g_{k}\right) .
$$

Denoting by $\Pi_{U F}$ the set of uncoordinated FDMA strategies, the outage function can now be derived as follows:

$$
\begin{aligned}
& 1-\mathcal{E}^{\Pi_{U F}}(\lambda, \mu, \mathcal{R})=\frac{\max _{\Pi_{U F}} \mathbb{E}_{K,\left\{g_{k}\right\}}\left(K_{s}\right)}{\lambda \tau_{s}} \\
& \stackrel{(a)}{=} \frac{\max _{B} \mathbb{E}_{K,\left\{g_{k}\right\}}\left\{\widetilde{B} \times \mathbf{1}\left[\widetilde{\mathcal{R}}_{f}\left(g_{k}, \mu, B\right) \geq \mathcal{R}\right]\right\}}{\lambda \tau_{s}} \\
& \stackrel{(\text { b) }}{=} \frac{\max _{B}\left\{\mathbb{E}_{K}[\widetilde{B}] \times \mathbb{E}_{\left\{g_{k}\right\}} \mathbf{1}\left[\widetilde{\mathcal{R}}_{f}\left(g_{k}, \mu, B\right) \geq \mathcal{R}\right]\right\}}{\lambda \tau_{s}} \\
& \stackrel{(c)}{=} \frac{\max _{B}\left\{\lambda \exp (-\lambda / B) \times \mathbb{P}\left[\widetilde{\mathcal{R}}_{f}\left(g_{k}, \mu, B\right) \geq \mathcal{R}\right]\right\}}{\lambda \tau_{s}} \\
& \stackrel{(d)}{=} \frac{\max _{B}\left\{\lambda \exp (-\lambda / B) \times \mathbb{P}\left[g_{k} \geq \frac{2^{B \mathcal{R}}-1}{B \mu}\right]\right\}}{\lambda \tau_{s}}
\end{aligned}
$$

where $\widetilde{B}$ in $(a)$ is the number of frequency bins with exactly one arrival, which is a function of total number of arrivals in the slot $(K)$ and the number of partitions $(B),(a)$ follows from the fact that for a successful transmission, the user should arrive in one of the $\widetilde{B}$ bins and should have a strong enough channel to achieve rate $\mathcal{R},(b)$ follows from the independence of these two events, $(c)$ follows from the fact that the number of arrivals in each subband is Poisson distributed as stated above, and $(d)$ follows from (34).

\section{NumericAl RESUlts}

For the numerical results, we assume that the reference SNR is $\mu=0 \mathrm{~dB}$. This would be obtained, for example, with a device transmitting with $10 \mathrm{dBm}(10 \mathrm{~mW})$ power over $1 \mathrm{MHz}$ bandwidth, a noise power spectral density of $-174 \mathrm{dBm} / \mathrm{Hz}$, a receiver noise figure of $5 \mathrm{~dB}$, a receiver antenna gain of $14 \mathrm{~dB}$, a 3.76 pathloss exponent, a $128 \mathrm{~dB}$ pathloss intercept at $1000 \mathrm{~m}$, and a reference distance of $1360 \mathrm{~m} \mathrm{[11].} \mathrm{Assume} \tau_{s}=1 \mathrm{sec}$.

Fig. 2 shows the outage probability $\epsilon$ versus the user rate $\mathcal{R}$ for a given arrival rate of $\lambda=16$ users per second. The outages for the optimal and FDMA strategies are given respectively by (17) and (39). We note that the for the given outage probability, the common rate supported by the optimal strategy is significantly higher than the FDMA strategy. In particular, in the regime of interest where the outage probability is $\epsilon=0.1$, the rate using the optimal strategy is $7 \mathrm{x}$ the FDMA rate.

Fig. 3 presents the corresponding throughput for the two transmission options computed using (12) for $\lambda=16$ arrivals per second. For the case of the uncoordinated optimal 


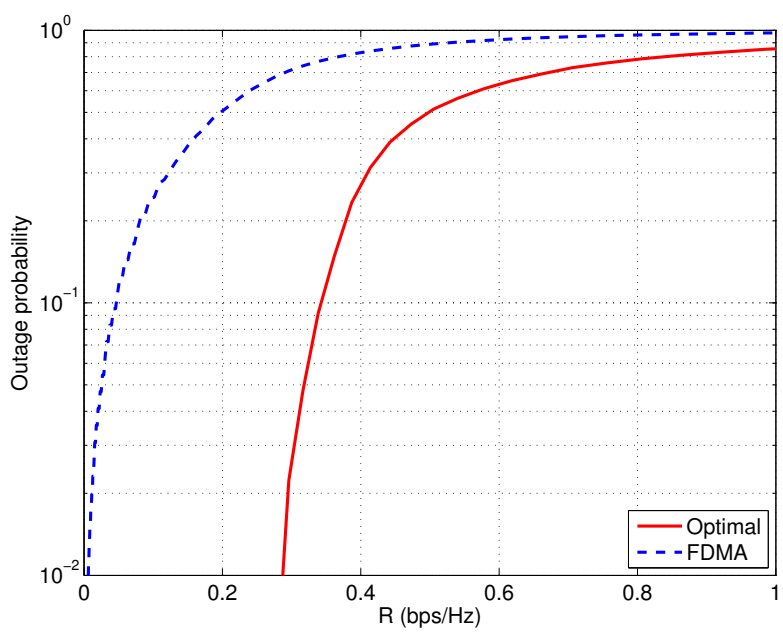

Fig. 2. Outage $\epsilon$ versus rate per user $\mathcal{R}$, for $\lambda=16$ and $\mu=0 \mathrm{~dB}$.

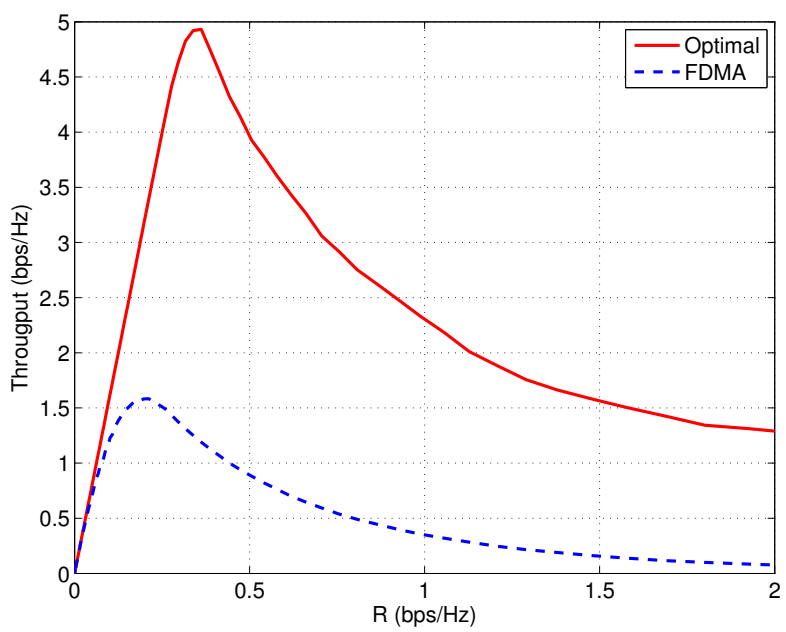

Fig. 3. Throughput $\mathcal{S}$ versus rate per user $\mathcal{R}$, for $\lambda=16$ and $\mu=0 \mathrm{~dB}$.

transmission, the maximum throughput is achieved when $\mathcal{R}=$ 0.35 , and the corresponding outage, obtained from Fig. 2, is $\epsilon=0.20$. For the FDMA case, the maximum throughput is achieved at $\mathcal{R}=0.2$, which corresponds to the outage $\epsilon=$ 0.50 in Fig. 2. Since the practical system would not tolerate such a high outage, this motivates us to consider the throughputs subject to some maximum outage, say $\epsilon_{\max }=0.1$. With this maximum outage, the rate-maximized throughput given by (13) for $\lambda=16$ is $0.7 \mathrm{bps} / \mathrm{Hz}$ and $4.9 \mathrm{bps} / \mathrm{Hz}$ for FDMA and optimal transmission, respectively.

Fig. 4 shows the rate-maximized throughput as a function of $\lambda$ with $\epsilon_{\max }=0.1$. As the arrival rate $\lambda$ increases, the rate achieved per user decreases, but the overall ratemaximized throughput increases for both the FDMA and optimal strategies. The gain in rate-maximized throughput of the optimal strategy versus FDMA increases logarithmically with $\lambda$. While the gain is $7 \mathrm{x}$ with $\lambda=16$, it is $10 \mathrm{x}$ with $\lambda=200$.

\section{CONCLUSION}

In this paper, we incorporated decoding techniques of coordinated MAC to random access and obtained a new fun-

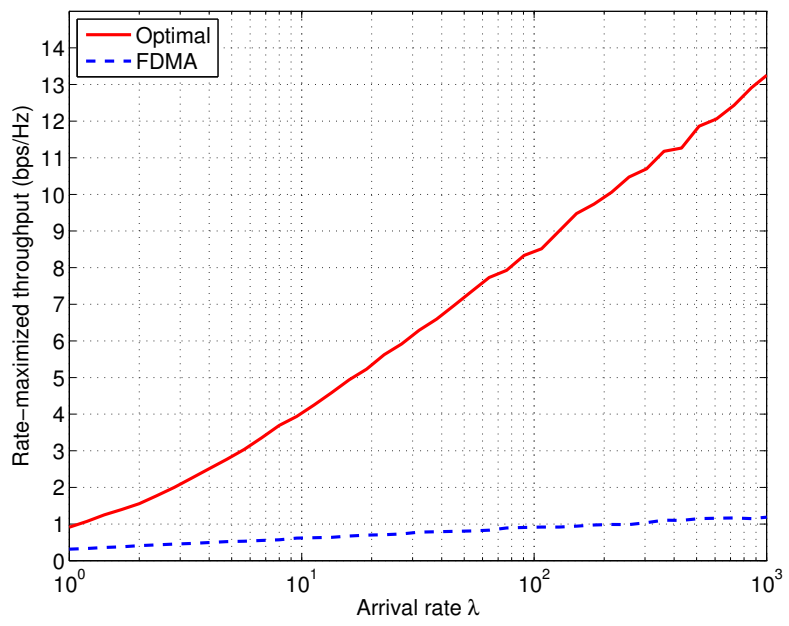

Fig. 4. Rate-maximized throughput versus arrival rate $\lambda$, where for a given $\lambda$, throughput is constrained such that the outage is no more than $\epsilon_{\max }=0.1$.

damental result characterizing the throughput performance of optimal uncoordinated random access transmission with joint decoding. The proposed scheme is shown to provide order of magnitude throughput improvement over slotted ALOHA. This work has numerous extensions. From information theoretic perspective, it is important to extend it to the case where each user has knowledge of its own channel and can thus perform power control in a distributed way. From cellular systems perspective, it is important to extend this study to multi-cell scenarios. For delay-tolerant applications, it is important to study the effect of retransmissions on the throughput performance.

\section{REFERENCES}

[1] S. Sesia, I. Toufik, and M. Baker, Eds., LTE: The UMTS Long Term Evolution. John Wiley and Sons, 2009.

[2] H. S. Dhillon, H. C. Huang, H. Viswanathan, and R. A. Valenzuela, "On resource allocation for machine-to-machine (M2M) communications in cellular networks," in Proc., IEEE Globecom Workshops, Anaheim, CA, Dec. 2012.

[3] Y. Jou, R. Attar, S. Ray, J. Ma, and X. Zhang, "M2M over CDMA2000 1x case studies," in Proc., IEEE Wireless Communications and Networking Conf. (WCNC), Cancun, Mexico, Mar. 2011, pp. 1546 - 1551.

[4] A. Ephremides and B. Hajek, "Information theory and communication networks: An unconsummated union," IEEE Trans. on Info. Theory, vol. 44, no. 6, pp. 2416 - 2434, Oct. 1998.

[5] P. Minero, M. Franceschetti, and D. Tse, "Random access: An information-theoretic perspective," IEEE Trans. on Info. Theory, vol. 58, no. 2, pp. 909 - 930, Feb. 2012.

[6] Study on RAN improvements for Machine-Type Communications, 3GPP TR 37.868, 2010.

[7] D. Slepian, "Bounds on communications," The Bell System Technical Journal, vol. 42, no. 3, pp. 681-707, May 1963.

[8] T. M. Cover and J. A. Thomas, Elements of information theory. New York: Wiley-Interscience, 1991.

[9] H. S. Dhillon, H. C. Huang, H. Viswanathan, and R. A. Valenzuela, "Power-efficient system design for cellular-based machine-to-machine communications," IEEE Tran. Wireless Communications, to appear. Available online: arxiv.org/abs/1301.0859.

[10] J. F. C. Kingman, Poisson Processes. Oxford University Press, 1993

[11] H. Huang, C. B. Papadias, and S. Venkatesan, MIMO Communication for Cellular Networks. Springer, 2012. 